\title{
Characterization and Optimization of the Novel Transient Receptor Potential Melastatin 2 Antagonist tatM2NXפ
}

\author{
이. Cruz-Torres, D.S. Backos, and P.S. Herson \\ Departments of Pharmacology (I.C.-T., P.S.H.) and Anesthesiology (P.S.H.) and Neuronal Injury \& Plasticity Program (I.C.-T., \\ P.S.H.), University of Colorado School of Medicine, Aurora, Colorado; and Department of Pharmaceutical Sciences, University of \\ Colorado Skaggs School of Pharmacy and Pharmaceutical Sciences, Aurora, Colorado (D.S.B.)
}

Received June 14, 2019; accepted November 18, 2019

\section{ABSTRACT}

Transient receptor potential melastatin 2 (TRPM2) is a calciumpermeable channel activated by adenosine diphosphate ribose metabolites and oxidative stress. TRPM2 contributes to neuronal injury in the brain caused by stroke and cardiac arrest among other diseases including pain, inflammation, and cancer. However, the lack of specific inhibitors hinders the study of TRPM2 in brain pathophysiology. Here, we present the design of a novel TRPM2 antagonist, tatM2NX, which prevents ligand binding and TRPM2 activation. We used mutagenesis of tatM2NX to determine the structure-activity relationship and antagonistic mechanism on TRPM2 using whole-cell patch clamp and Calcium imaging in human embryonic kidney 293 cells with stable human TRPM2 expression. We show that tatM2NX inhibits over $90 \%$ of TRPM2 channel currents at concentrations as low as $2 \mu \mathrm{M}$. Moreover, tatM2NX is a potent antagonist with an $\mathrm{IC}_{50}$ of $396 \mathrm{nM}$. Our results from tatM2NX mutagenesis indicate that specific residues within the tatM2NX C terminus are required to confer antagonism on TRPM2. Therefore, the peptide tatM2NX represents a new tool for the study of TRPM2 function in cell biology and enhances our understanding of TRPM2 in disease.

\section{SIGNIFICANCE STATEMENT}

TatM2NX is a potent TRPM2 channel antagonist with the potential for clinical benefit in neurological diseases. This study characterizes interactions of tatM2NX with TRPM2 and the mechanism of action using structure-activity analysis.

\section{Introduction}

The transient receptor potential melastatin 2 (TRPM2) is a nonselective cation channel from the TRP family. At physiologic membrane potentials, activation of TRPM2 results in influx of sodium and calcium (Ca2+) into the cell. TRPM2 forms tetramers composed of six transmembrane domains with the $\mathrm{N}$ terminus (Nterm) and C terminus (Cterm) facing the intracellular milieu. The Cterm contains a unique Nudix hydrolase 9 homology domain (NUDT9-H) required for adenosine diphosphate ribose (ADPR) binding and activation, absent in other TRP family members (Tong et al., 2006; Hantute-Ghesquier et al., 2018). TRPM2 was initially described as a channel/enzyme (chanzyme) because of the presence of the NUDT9-H domain. However, subsequent work has demonstrated that the C-terminal NUDT9-H domain of TRPM2 lacks enzymatic activity (Perraud et al., 2003; Iordanov et al., 2016). The NUDT9-H domain of

This work was supported by the National Institutes of Health Grant T32GM007635 (Pharmacology Training Grant) and Grant R01NS092645 (to P.S.H.).

https://doi.org/10.1124/mol.119.117549. org.

S This article has supplemental material available at molpharm.aspetjournals.

ABBREVIATIONS: ADPR, adenosine diphosphate ribose; ADPR $_{\mathrm{f}}$, final ADPR current; $\mathrm{ADPR}_{\mathrm{i}}$, initial ADPR current; BBB, blood-brain barrier; $\mathrm{BSA}$, bovine serum albumin; Cterm, C terminus; CTZ, clotrimazole; Dox, doxycycline; GSK3 $\beta$, glycogen synthase kinase 3 beta; HEK, human embryonic kidngey; HEPES, 4-(2-hydroxyethyl)-1-piperazinepropanesulfonic acid; HIV, human immunodeficiency virus; MD, molecular dynamic; Nterm, N terminus; NUDT9-H, Nudix hydrolase 9 homology domain; PVDF, polyvinylidene difluoride; TRPM2, transient receptor potential melastatin 2; YASARA, Yet Another Scientific Artificial Reality Application.
TRPM2 is essential for intra- and interface interactions that regulate TRPM2 channel activation by ADPR (Huang et al., 2018; Wang et al., 2018). Intracellular $\mathrm{Ca}^{2+}$ serves as a coagonist, modulating channel activity in the presence of ADPR (Herson et al., 1997, 1999; Perraud et al., 2001; Inamura et al., 2003; McHugh et al., 2003; Kühn and Lückhoff, 2004; Heiner et al., 2006; Olah et al., 2009; Tóth et al., 2015; Fliegert et al., 2017a,b; Yu et al., 2017). Consistent with these physiologic observations, the recently solved human TRPM2 channel structure indicates an ADPR binding site in the Cterm that appears to interact with the Nterm upon opening and leads to structural changes in a calcium-dependent "primed" state (Huang et al., 2018; Wang et al., 2018).

TRPM2 channels have been implicated in several physiological and pathophysiological conditions in multiple organs (Inamura et al., 2003; Smith et al., 2003; Fonfria et al., 2004, 2006; Kraft et al., 2004; Lange et al., 2009; Haraguchi et al., 2012; Verma et al., 2012; Alim et al., 2013; Shimizu et al., 2013; Gelderblom et al., 2014; Hoffman et al., 2015; Jang et al., 2015; Park et al., 2016; Tan and McNaughton, 2016; Andoh et al., 2019; Li and Jiang, 2019). TRPM2 channels are highly expressed in the brain, found in neurons and microglia in the cortex, hippocampus, striatum, brainstem, and others 
(Fonfria et al., 2006; Olah et al., 2009; Chung et al., 2011). TRPM2 channels are activated following oxidative stress, and the most well-characterized role for these channels is a cell death mediator following oxidative stress due to excessive $\mathrm{Ca}^{2+}$ influx and consequent cell death (Fonfria et al., 2004; Perraud et al., 2005; Bai and Lipski, 2010). However, the lack of specific TRPM2 channel antagonists has hindered the research regarding the role of TRPM2 channels in brain function, with most data coming from cell culture experiments or global TRPM2 channel genetic ablation in mice. Thus far, most described TRPM2 pharmacological inhibitors are not specific to TRPM2 channels, including antifungals, flufenamic acid, fenamate nonsteroidal anti-inflammatories, 2-aminoethoxydiphenyl borate, and natural compounds with moderate to high potency (Hill et al., 2004; Chen et al., 2012; Starkus et al., 2017; Zhang et al., 2018). The TRPM2 inhibitor JNJ-28583113 is a recently described inhibitor that appears promising, with nanomolar potency when applied to the extracellular surface of TRPM2 channels. However, it is limited by stability difficulties in the brain (Fourgeaud et al., 2019). In contrast, we recently reported that the inhibitor, tatM2NX, reduces ischemic injury when administered following focal cerebral ischemia (Shimizu et al., 2016) and global cerebral ischemia (Dietz et al., 2019) in vivo, providing evidence for clinical benefit. Therefore, the aim of this study is to characterize the TRPM2 channel inhibitor tatM2NX, a peptide designed to interact with the ADPR binding site on the NUT9-H domain.

In this structure-activity relationship study, we show that tatM2NX is an antagonist of human TRPM2 channels using whole-cell patch clamp and calcium imaging in human embryonic kidney (HEK) 293 cells. Mutagenesis of tatM2NX reveals that the mechanism of action results from tatM2NX C-terminal interactions with TRPM2 channels. Ultimately, tatM2NX is a potent pharmacological tool to disentangle TRPM2 function in cellular physiology and neurological diseases.

\section{Materials and Methods}

Protein Structure Prediction and Molecular Modeling. All molecular modeling studies were conducted using Biovia Discovery Studio 2018 (Biovia, Inc., Sand Diego, CA; www.3dsbiovia.com) and Yet Another Scientific Artificial Reality Application (YASARA) Structure 18.4 (YASARA Biosciences GmbH, Vienna, Austria; www.yasara. org). Structural coordinates for the human TRPM2 ion channel (Wang et al., 2018) were downloaded from the Protein Data Bank (www.rcsb. org, accession: 6MIX). Ab initio prediction of the secondary and tertiary structures for the wild-type, truncated, and mutant peptides was performed using the online QUARK server (https://zhanglab. ccmb.med.umich.edu/QUARK/) (Xu and Zhang, 2012, 2013). The top five predicted peptide structures for each designed peptide were subjected to 1 nanosecond of explicit solvent-based molecular dynamic (MD) simulation utilizing the YASARA2 force field (Krieger et al., 2006, 2009, 2012; Krieger and Vriend, 2015), which combines the Amber (ff14SB) force field (Maier et al., 2015) with self-parameterizing knowledge-based potentials (Krieger et al., 2002), to refine each of the predicted peptide structures. The snapshots from the resulting trajectories were assessed using the WHAT_IF and WHAT_CHECK (Vriend, 1990; Hooft et al., 1996) structure validation tools to quantitatively evaluate the overall quality of each predicted structure, with the highest scoring structure for each peptide selected for further analysis. The ZDOCK (Chen et al., 2003), ZRANK (Pierce and Weng, 2007), and RDOCK (Li et al., 2003) algorithms were employed within Discovery Studio to predict the most likely protein-peptide complexes and refine their respective intermolecular interactions, as we have described previously (Ryan et al., 2012; Smith et al., 2018). To test the stability of the predicted interactions, we first removed the transmembrane domain of the TRPM2 subunit (residues 697-1165), and the top scoring complex for each peptide was placed in a simulation cell under periodic boundary conditions, filled with water, $0.9 \% \mathrm{NaCl}$, and counter ions, $\mathrm{pH} 7.4$, at a temperature of $298 \mathrm{~K}$ (Krieger et al., 2004). The main MD simulation was run for 5 nanoseconds using the Amber (ff14SB) force field (Maier et al., 2015) with GAFF (Wang et al., 2004)/AM1BCC (Jakalian et al., 2002) parameters, particle mesh Ewald summation, an 8.0-Å cutoff for nonbonded forces, a 5-femtosecond time step, and LINCS-constrained hydrogen atoms (Hess et al., 1997) and kept at constant pressure and temperature (the NPT ensemble), as described previously (Krieger and Vriend, 2015). Figures were generated using Lightwave 2019 (NewTek Inc., Burbank, CA; www.lightwave3d.com) and Marmoset Toolbag 3.07 (Marmoset, LLC, Portland, OR; www.marmoset.co).

Cell Culture. Doxycycline-inducible N-terminal FLAG-TRPM2expressing HEK293 cells, provided by Anne L. Perraud (University of Colorado Anschutz Medical Campus, CO), were cultured as previously described (Perraud et al., 2001; Shimizu et al., 2016). Briefly, cells were grown in Advanced Dulbecco's modified Eagle's medium containing $10 \%$ fetal bovine serum, 2 mM GlutaMAX (Life technologies, Carlsbad, CA), and MycoZap-Plus (Lonza, Switzerland). Cell line authentication was confirmed as female human embryonic kidney cells, and mycoplasma contamination was negative (BioResources Core, University of Colorado, Anschutz Medical Campus, Aurora, CO). During growth, selection markers Zeocin $(1 \mu \mathrm{g} / \mathrm{ml})$ (Invitrogen, Carlsbad, CA) and Blastocidin-S $(0.4 \mu \mathrm{g} / \mathrm{ml})$ (Gibco, Carlsbad, CA) were used for the selective expression of tetracycline repressor and human TRPM2 in HEK293 cells until 80\%-90\% confluence. Cells were grown for up to 20 passages (P3-P20). For Western blot experiments, HEK293-derived cells were maintained in Zeocin and Blastocidin-S (uninduced cells) or in doxycycline $(1 \mu \mathrm{g} / \mathrm{ml}$ ) (induced cells) for 16-18 hours prior to protein lysate collection. For electrophysiology experiments, HEK293 cells were seeded on 12-mm glass coverslips at a density of 12,000 cells $/ \mathrm{ml}$ for $16-24$ hours for doxycycline-inducible human TRPM2 expression prior to experiments. For $\mathrm{Ca}^{2+}$ imaging experiments, HEK293 cells were seeded on Mattek glass bottom dishes (Mattek Corporation, Ashland, MA) at a density of 25,000 cells $/ \mathrm{ml}$ for $16-24$ hour prior to experiments.

Western Blot. HEK293 cells were collected 16-18 hours after induction via centrifugation at 3,000 rpm for 3 minutes, washed in PBS (1× PBS, pH 7.4), and lysed for 10 minutes using neuronal protein extraction reagent (Thermo Scientific, Rockford, IL). For glycogen synthase kinase 3 beta (GSK3 $\beta$ ) expression and phosphorylation, HEK293 cells were preincubated with tatM2NX for 30 minutes to 4 hours followed by $250 \mu \mathrm{M} \mathrm{H}_{2} \mathrm{O}_{2}$ stimulation (10 minutes), and protein lysates were collected immediately. Lysates were centrifuged at $12,000 \mathrm{rpm}$ for 15 minutes, and supernatant was collected for protein quantification. Protein samples (15-20 $\mu \mathrm{g})$ were resolved using SDSPAGE and transferred in polyvinylidene difluoride (PVDF) membranes ( $\beta$ actin, 50 minutes; FLAG-TRPM2, 90 minutes; GSK3 $\beta, 50$ minutes). PVDF membranes were blocked in $5 \%$ bovine serum albumin (BSA) for 1 hour and incubated overnight at $4^{\circ} \mathrm{C}$ in primary antibody. Human TRPM2 expression was assessed with mouse anti-FLAG (1:1000, F1804; Sigma, St. Louis, MO) (Brizzard et al., 1994) and normalized to mouse anti- $\beta$ actin peroxidase (1:10,000, A3854; Sigma). GSK3 $\beta$ phosphorylation and expression were assessed with rabbit anti-pGSK3 or GSK3 $\beta$ (1:1000, 9323S; and 1:1000, 12456S; Cell Signaling Technology, Danvers, MA). All membranes were washed three times and incubated in secondary horseradish peroxidase-conjugated goat anti-mouse or goat anti-rabbit antibodies (1:10,000, 115-035-174, or 115-035-003; ImmunoResearch Laboratories, West Grove, PA; or 1: 10,000, 31460; ThermoFisher Scientific) for 1 hour at room temperature. Western blot bands were detected using the SuperSignal West Femto Maximum Sensitivity Substrate (34096; ThermoFisher Scientific) and imaged with BioRad ChemiDoc MP Imaging System (Hercules, CA). 
Immunocytochemistry. Doxycycline-inducible human TRPM2expressing HEK293 cells (16-18 hours) were fixed with $4 \%$ paraformaldehyde for 10 minutes on ice, washed in $1 \times$ PBS, permeabilized using $0.3 \%$ Triton X-100 dissolved in $1 \times$ PBS for 10 minutes at room temperature, and blocked in $4 \%$ bovine serum albumin overnight at $4^{\circ} \mathrm{C}$. For biotin-tatM2NX detection, HEK293 cells were incubated with the peptide for 1 hour prior to fixation. Primary antibody (mouseanti-FLAG, 1:1000, F1804; Sigma) was incubated for 2 hours at room temperature in $2.5 \% \mathrm{BSA} / \mathrm{PBS}$ and secondary antibody (594conjugated streptavidin, 1:1000, 016-540-084; or 488-donkey-antimouse, 1:1000, 715-545-150; ImmunoResearch Laboratories) for 1 hour at room temperature in $2.5 \%$ BSA/PBS. Then, coverslips were mounted using Prolong Gold Antifade Agent (Thermo Fisher Scientific). Images of HEK293 cells were taken at room temperature on an Olympus IX83 (Olympus Fluoview FV1200 Laser Scanning Confocal Microscope; Olympus Life Science, MA) using a $20 \times$ objective. Two individual observers analyzed all images.

Coimmunoprecipitation. Protein lysates $(500 \mu \mathrm{g})$ from doxycyclineinducible human TRPM2-expressing HEK293 cells (16-18 hours) containing an $\mathrm{N}$-terminal FLAG tag were incubated for 1 hour with $\mathrm{N}$-terminal biotin-tagged tatM2NX $(20 \mu \mathrm{g})$ following the addition of prewashed streptavidin-conjugated agarose (60 $\mu$ l, 16-126; Sigma Aldrich) at $4^{\circ} \mathrm{C}$ on a rocker. Samples were washed three times with phosphate buffer (100 mM NaCl, pH 7.4). Purified biotin-tatM2NX complexes with FLAG-TRPM2 were boiled at $95^{\circ} \mathrm{C}$ for 5 minutes in Laemmli dye (40-50 $\mu \mathrm{l}$; BioRad). Bands were resolved using Precast Miniprotean gradient gels (4\%-20\% acrylamide; BioRad), cut in half, and transferred for 30 minutes for biotin-tatM2NX $(5 \mathrm{kDa})$ and 90 minutes for FLAG-TRPM2 $(170 \mathrm{kDa})$ in PVDF membranes $(0.22$ or $45 \mu \mathrm{m})$. FLAG-TRPM2 was probed using primary mouse antiFLAG M2 antibody (F1804; Sigma Aldrich) and secondary horseradish peroxidase-conjugated goat anti-mouse (1:10,000, 115-035-003 or 111-035-003; ImmunoResearch Laboratories). See Western Blot for membrane development.

Peptide Synthesis. TatM2NX and derivatives were synthetized commercially with $>95 \%$ purity (Chi Scientific, Maynard, MA). All peptides contained a cell-permeant $\mathrm{N}$ terminus tat-human immunodeficiency virus (HIV) sequence (YGRKKRRQRRR) fused to the M2NX sequence variations (Table 1). For coimmunoprecipitation, biotin was conjugated to tatM2NX.

Electrophysiology. All recordings were performed in HEK293 cells, and currents were amplified with Axopatch 200B (Axon Instruments), digitized with DigiData1550B, and controlled using pClamp10.7 software (Molecular Devices, CA). Signals were filtered at $5 \mathrm{kHz}$ and digitized at $1 \mathrm{kHz}$. Glass borosilicate electrodes (3.5-5 M $\Omega$ ) were used to record human TRPM2 currents activated by $+40 \mathrm{mV}$ voltage step from $0 \mathrm{mV}$ in whole-cell voltage clamp configuration. The protocol was chosen to produce an outward current and therefore reduce TRPM2-mediated calcium influx and subsequent cell death. All experiments were performed with 4-(2-hydroxyethyl)-1-piperazinepropanesulfonic acid (HEPES)-buffered saline containing, in millimolars, $140 \mathrm{NaCl}, 2.5 \mathrm{KCl}, 10 \mathrm{HEPES}, 5$ glucose, $1 \mathrm{MgCl}_{2}$, and 1 $\mathrm{CaCl}, \mathrm{pH}$ 7.4. The internal solution contained, in millimolars, 145 K-gluconate, 0.05 EGTA (for physiologic buffering of $\mathrm{Ca}^{2+}$ at $100-200 \mathrm{nM}), 1 \mathrm{MgCl}_{2}$, and $10 \mathrm{HEPES}, \mathrm{pH}$ 7.3. For antagonism, the internal solution contained $100 \mu \mathrm{M}$ ADPR (A0752; Sigma Aldrich) and $0,0.05,0.15,0.3,0.5,2,5$, or $10 \mu \mathrm{M}$ tatM2NX. The inclusion of tatM2NX in the internal solution allows access of controlled concentration to the intracellular ADPR binding site on TRPM2; however, no solution exchange is possible to assess reversibility. For competitive antagonism, the internal solution contained $500 \mu \mathrm{M}$ ADPR and 0 or $2 \mu \mathrm{M}$ tatM2NX. For structure-activity relationship experiments, the internal solution contained $100 \mu \mathrm{M}$ ADPR and $2 \mu \mathrm{M}$ peptide (tatWV-AA, tat Cterm, tat Nterm). We added clotrimazole (CTZ, $20 \mu \mathrm{M}$, C6019-G; Sigma) after steady-state inhibition as a positive control. Exclusion criteria were based on access resistance $R_{a}<15 \mathrm{M} \Omega$ and remaining leak current $<350 \mathrm{pA}$ after CTZ inhibition.

$\mathbf{C a}^{2+}$ Imaging. Live cell imaging of HEK293 cells was performed at room temperature on an Olympus IX83 (Olympus Fluoview FV1200 Laser Scanning Confocal Microscope; Olympus Life Science) using a $10 \times$ objective. For all imaging experiments in this study, controls and peptides were tested each experimental day to account for passage differences. All images were processed using FIJI Software (Rueden et al., 2017). Each plate was washed three times with HEPES-buffered saline (see Electrophysiology above). A total volume of $2 \mathrm{ml}$ was added to each plate with $5 \mu \mathrm{M}$ of the $\mathrm{Ca}^{2+}$ indicator Fluo5F, acetoxymethyl ester (Invitrogen, Eugene, OR), with or without $2 \mu \mathrm{M}$ peptide (tatWV-AA, tat Cterm, tat Nterm), or $20 \mu \mathrm{M} \mathrm{CTZ}$ and incubated at $37^{\circ} \mathrm{C}$ for $40-50$ minutes prior to the experiments. Then, plates were washed, and fresh solution was added with or without drugs. Excitation illumination was delivered every 10 seconds for 20 minutes. After a 1-minute baseline, TRPM2 activity was stimulated using $250 \mu \mathrm{M} \mathrm{H}_{2} \mathrm{O}_{2}$, and fluorescence was recorded for 20 minutes. Cells were incubated with an antagonist prior to exposure to $\mathrm{H}_{2} \mathrm{O}_{2}$, which activates TRPM 2 channels, resulting in cell death within 30-60 minutes because uncontrolled $\mathrm{Ca}^{2+}$ influx through activated TRPM2 channels is toxic to cells. Therefore, we chose to image for 20 minutes to assess the inhibition of each peptide, and we were unable to assess reversibility.

Statistical Analysis. For analysis of steady-state inhibition of TRPM2 currents, a paired or independent samples $t$ test was performed with statistical significance of $P<0.05$ for $n \geq 4-10$. Potency $\left(\mathrm{IC}_{50}\right)$ was determined using a nonlinear regression analysis (log of inhibitor concentration versus normalized response) equation: $\mathrm{Y}=100 /(1+$ $\left.10^{\wedge}((\mathrm{X}-\operatorname{LogIC50}))\right) ; \mathrm{X}=\log$ of concentration, $\mathrm{Y}=$ normalized response (current density $\mathrm{pA} / \mathrm{pF}$ ), using Graph Pad Prism version 8.0.2 for Windows (GraphPad Software, La Jolla, CA, www.graphpad.com). For $\mathrm{Ca}^{2+}$ imaging, changes in $\mathrm{F} / \mathrm{F}_{0}(n=6-12$ individual cells per plate) were used to compare groups after normalizing to background fluorescence. Individual regions of interest were drawn for each cell and analyzed using the Time Series Analyzer plugin in FIJI Software (Rueden et al., 2017). Area under the curve for each group was determined, and groups were compared with control $\mathrm{H}_{2} \mathrm{O}_{2}$ using one-way ANOVA with Dunnett's post hoc test for multiple group comparison. Statistical significance was determined as $P<0.05$, and all groups were represented as means \pm S.D.

\section{Results}

Modeling for tatM2NX Antagonism on TRPM2. We recently showed that the peptide tatM $2 \mathrm{NX}$ is a TRPM2 antagonist with selective neuroprotective effects in a mouse model of focal ischemia (stroke) (Shimizu et al., 2016) and global ischemia (cardiac arrest and cardiopulmonary resuscitation) (Dietz et al., 2019). To characterize the mechanism of TRPM2 inhibition by tatM2NX, we performed molecular modeling (MD) to allow prediction of tatM2NX interaction/ inhibition of activity and potential key residues responsible for efficacy. $\mathrm{Ab}$ initio prediction of the secondary and tertiary

TABLE 1

Description of peptides

\begin{tabular}{lcl}
\hline Peptide & Concentration $(\mu \mathrm{M})$ & Description \\
\hline TatM2NX & $0.05-10$ & Cell permeable, parent peptide YGRKKRRQRRRGSREPGEMLPR KLKRVLRQEFWV \\
TatWV-AA & 2 & Parent peptide, residues mutated to alanine YGRKKRRQRRRGSREPGEMLPR KLKRVLRQEFAA \\
Tat Cterm & 2 & Truncated peptide YGRKKRRQRRRKLKRVLRQEFWV \\
Tat Nterm & 2 & Truncated peptide YGRKKRRQRRRGSREPGEMLPR \\
\hline
\end{tabular}


structure of the parent tatM2NX peptide was predicted to adopt a single, $\alpha$ helical structure that remained stable during MD-based refinement and analysis (Fig. 1A; Table 1).

Protein-peptide complex prediction for tatM2NX indicated that the top-scoring cluster for the peptide involved direct interaction with the human ADPR binding site (Fig. 1B), which suggests occlusion of this site and the resulting inhibition of ADPR binding as a potential mechanism for the observed functional inhibition of TRPM2 in vitro. To test the stability of the predicted complex, we performed a 5-nanosecond MD-based simulation in the presence of an explicit solvent. The TRPM2tatM2NX complex remained stable throughout the simulation and appeared to settle further into the ADPR binding site and enhance the number of favorable interactions between tatM2NX and TRPM2 compared with the initial predicted complexes (Fig. 1C; Table 2). At the end of the MD simulation period, tatM2NX made several favorable intermolecular interactions, including salt bridges with Arg1280 and Arg1433 as well as an extensive network of intermolecular hydrogen bonds and hydrophobic interactions (Table 2). TatM2NX also appeared to form a wedge-like conformation, with the $\mathrm{C}$ terminus portion interacting with the ADPR binding site and the Tat-HIV tag ( $\mathrm{N}$ terminus) interacting with the opposite side the TRPM2 monomer (Fig. 1C, right).

Human TRPM2 Channels are Expressed and Functional in HEK293 Cells. We measured TRPM2 protein expression and function in HEK293 cells. Human TRPM2 (N-terminal FLAG tag) is expressed in HEK293 cells after doxycycline treatment (16-18 hours) (Supplemental Fig. 1, $\mathrm{A}$ and $\mathrm{B})$. To validate human TRPM2 channel function, we performed whole-cell patch-clamp experiments in HEK293 cells with ADPR present in the pipette solution to stimulate TPRM2-mediated currents. We found that human TRPM2 exhibited large initial current density [initial ADPR current $\left(\mathrm{ADPR}_{\mathrm{i}}\right), 184.4 \pm 63.73 \mathrm{pA} / \mathrm{pF}, n=5$ ] exclusively under doxycycline treatment (Fig. 2A; Supplemental Fig. 1C). We observed significant rundown of TRPM2 initial current density [final $\mathrm{ADPR}$ current $\left(\mathrm{ADPR}_{\mathrm{f}}\right), 86.45 \pm 46.68 \mathrm{pA} /$ $\mathrm{pF}, n=5 ; P<0.05]$, which reached steady-state levels after 2-3 minutes, as previously described (Tóth and Csanady, 2012) (Supplemental Fig. 1C). Therefore, subsequent experimental analysis was performed using the steady-state current density at 3 minutes as the active TRPM2 current $\left(A D P R_{f}\right)$. Multiple control experiments were performed to confirm that the ADPR-induced current in HEK293 cells were carried by TRPM2 channels. Importantly, the TRPM2 channel pore blocker clotrimazole $(20 \mu \mathrm{M} \mathrm{CTZ})$ abolished ADPR currents $(6.359 \pm 3.666 \mathrm{pA} / \mathrm{pF}, n=5 ; P<0.05)$ (Supplemental Fig. 1C). Also, uninduced (no-doxycycline treatment) cells lacked TRPM2 expression or activity $(0.58 \pm 0.765 \mathrm{pA} / \mathrm{pF}$ vs. $0.09 \pm$ $1.67 \mathrm{pA} / \mathrm{pF}, P>0.05$ ) (Supplemental Fig. 1, A and D). These results validate TRPM2 expression and function in doxycyclinetreated HEK293 cells.

TatM2NX is a Potent TRPM2 Antagonist. To characterize tatM2NX as an antagonist, we performed a doseresponse study. To achieve a known concentration of ligand and antagonist within each HEK293 cell, we used whole-cell patch clamp with $100 \mu \mathrm{M}$ ADPR and tatM2NX added to the internal solution (pipette), allowing them to freely dialyze into the cell once whole-cell access was obtained. Our results show a concentration-dependent decrease in TRPM2 current density. We observed approximately $75 \%$ inhibition of initial TRPM2 current at $0.5 \mu \mathrm{M}$ tatM2NX $(37.82 \pm 53.19 \mathrm{pA} / \mathrm{pF}, n=5$; $P<0.05)$ and approximately $90 \%$ inhibition at $2 \mu \mathrm{M}(10.27 \pm$ $9.52 \mathrm{pA} / \mathrm{pF}, n=7 ; P<0.05), 5 \mu \mathrm{M}(7.76 \pm 6.387 \mathrm{pA} / \mathrm{pF}, n=5$; $P<0.05)$, and $10 \mu \mathrm{M}$ tatM2NX $(13.17 \pm 13.36, n=4 ; P<0.05)$ (Fig. 2, A and B). This inhibition was reached within 6-8 minutes. No significant differences were observed for $0.15 \mu \mathrm{M}$ $(121.6 \pm 62.65 \mathrm{pA} / \mathrm{pF}, P>0.05)$ and $0.3 \mu \mathrm{M}(118.0 \pm 50.68 \mathrm{pA} /$ $\mathrm{pF}, P>0.05)$ tatM2NX compared with $0.05 \mu \mathrm{M}(142.2 \pm$ $90.81 \mathrm{pA} / \mathrm{pF}, n=7$ ) (Fig. 2B), which was similar to ADPR alone $\left(\mathrm{ADPR}_{\mathrm{f}}, 86.45 \pm 46.68 \mathrm{pA} / \mathrm{pF}, n=5, P>0.05\right)$ (Supplemental Fig. 1E). A nonlinear regression method (log inhibitor concentration vs. normalized response) of the tatM2NX dose response estimated a potency $\left(\mathrm{IC}_{50}\right)$ of $3.96 \mathrm{e}-007 \pm 0.05868$ (396 nM) (Fig. 2C). These results suggest that tatM2NX is a potent antagonist of TRPM2 currents in the presence of ADPR.

To validate tatM2NX binding to TRPM2 as predicted in our molecular modeling, we performed coimmunoprecipitation using an N-terminal biotin tag on tatM2NX. To do this, we extracted whole-cell protein lysates from HEK293 cells and incubated protein lysates $(500 \mu \mathrm{g})$ with biotinylated tatM2NX $(20 \mu \mathrm{g})$. The peptide biotin-tatM2NX formed a complex with FLAG-TRPM2 when coimmunoprecipitated using streptavidin-conjugated

A

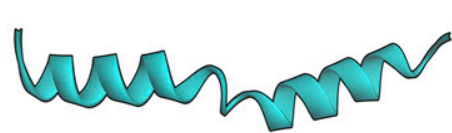

B
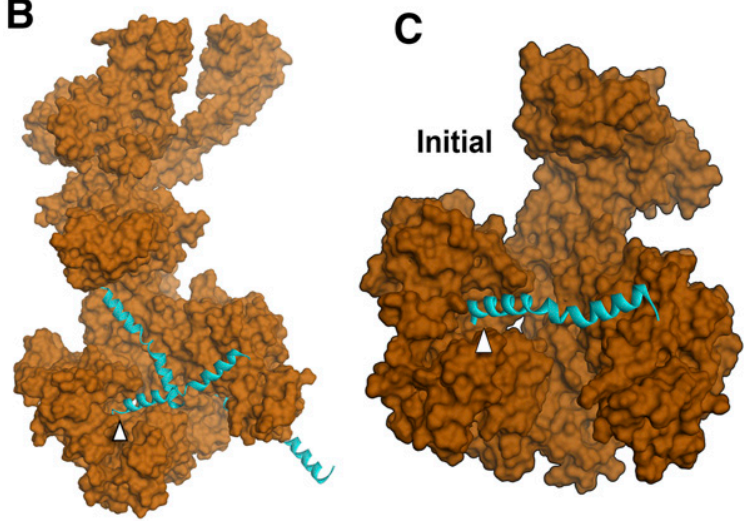

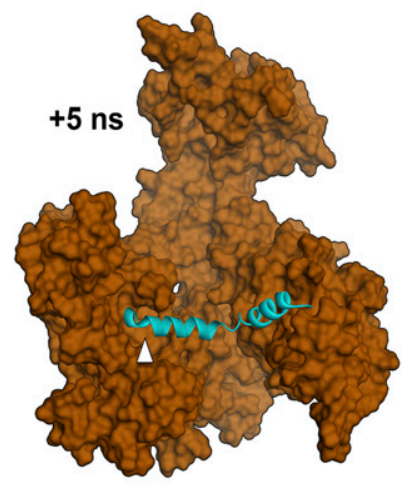

Fig. 1. Molecular modeling of tatM2NX with the human TRPM2 channel NUDT9-H domain. (A) Predicted secondary structure of tatM2NX after MD-based refinement. The peptide is oriented Nterm (left) to Cterm (right). (B) Top three predicted TPRM2-tatM2NX complexes; the arrowhead indicates the human ADPR binding site within the NUDT9-H domain. (C) Top scoring initial TPRM2-tatM2NX complex (left) and the same complex after 5 nanoseconds of MD simulation (right). 
TABLE 2

tatM2NX noncovalent interactions

Three-letter codes refer to amino acid.

\begin{tabular}{|c|c|c|}
\hline Interaction & Interaction Category & Interaction Type \\
\hline A:ARG1280:HH21 - E:GLU31:OE1 & Hydrogen bond; electrostatic & Salt bridge; attractive charge \\
\hline A:ARG1433:HH12 - E:VAL34:OXT & Hydrogen bond; electrostatic & Salt bridge; attractive charge \\
\hline A:ARG1433:HH22 - E:VAL34:OXT & Hydrogen bond; electrostatic & Salt bridge; attractive charge \\
\hline A:ARG1433:NH1 - E:GLU31:OE2 & Electrostatic & Attractive charge \\
\hline A:ARG1433:NH2 - E:VAL34:O & Electrostatic & Attractive charge \\
\hline E:ARG9:NH2 - A:GLU121:OE2 & Electrostatic & Attractive charge \\
\hline A:TYR1349:HH - E:VAL34:OXT & Hydrogen bond & Conventional hydrogen bond \\
\hline A:GLY1389:H - E:PHE32:O & Hydrogen bond & Conventional hydrogen bond \\
\hline A:SER1391:HG - E:GLU31:O & Hydrogen bond & Conventional hydrogen bond \\
\hline A:SER1391:HG - E:VAL34:O & Hydrogen bond & Conventional hydrogen bond \\
\hline A:LYS1401:HZ3 - E:GLU31:O & Hydrogen bond & Conventional hydrogen bond \\
\hline A:HIS1488:HE2 - E:TRP33:O & Hydrogen bond & Conventional hydrogen bond \\
\hline E:ARG6:HE - A:PHE108:O & Hydrogen bond & Conventional hydrogen bond \\
\hline E:ARG6:HH21 - A:PHE108:O & Hydrogen bond & Conventional hydrogen bond \\
\hline A:PRO1380:HD1 - E:GLN30:OE1 & Hydrogen bond & Carbon hydrogen bond \\
\hline A:SER1382:HB1 - E:GLN30:OE1 & Hydrogen bond & Carbon hydrogen bond \\
\hline A:SER1391:HB2 - E:VAL34:O & Hydrogen bond & Carbon hydrogen bond \\
\hline A:SER1391:HB2 - E:VAL34:OXT & Hydrogen bond & Carbon hydrogen bond \\
\hline E:ARG3:HA - A:GLN109:OE1 & Hydrogen bond & Carbon hydrogen bond \\
\hline E:LYS23:HE1 - A:PRO1248:O & Hydrogen bond & Carbon hydrogen bond \\
\hline E:LYS23:HE1 - A:ASN1249:O & Hydrogen bond & Carbon hydrogen bond \\
\hline E:ARG26:HD1 - A:LEU1381:O & Hydrogen bond & Carbon hydrogen bond \\
\hline A:CYS1250 - E:LEU20 & Hydrophobic & Alkyl \\
\hline E:ARG26 - A:LEU1381 & Hydrophobic & Alkyl \\
\hline A:PHE108 - E:ARG6 & Hydrophobic & Pi-Alkyl \\
\hline A:HIS1488 - E:VAL34 & Hydrophobic & Pi-Alkyl \\
\hline E:PHE32 - A:ARG1404 & Hydrophobic & Pi-Alkyl \\
\hline E:PHE32 - A:ILE1405 & Hydrophobic & Pi-Alkyl \\
\hline E:TRP33 - A:PRO1380 & Hydrophobic & Pi-Alkyl \\
\hline
\end{tabular}

A, TRPM2; E, peptide.

agarose (Fig. 3A). This binding interaction is specific to HEK293 cells expressing TRPM2 (lane 3) and absent in uninduced cells (lane 4) or beads without biotin-tatM2NX (lane 1). These results indicate that tatM2NX directly interacts with TRPM2.

We next assessed the ability of tatM2NX to inhibit TRPM2mediated dephosphorylation of GSK3 $\beta$ (activation) because this signaling pathway has recently been shown in cell culture (Fourgeaud et al., 2019) and brain slices (Dietz et al., 2019). TRPM2 stimulation with $\mathrm{H}_{2} \mathrm{O}_{2}(250 \mu \mathrm{M})$ activates GSK3 $\beta$ (dephosphorylation) in HEK293 cells (+control) compared with untreated cells (-control) (Fig. 3B). Preincubation with tatM2NX $(2 \mu \mathrm{M})$ for $2-4$ hours prevents GSK3 $\beta$ activation, indicating that tatM2NX inhibits TRPM2-mediated GSK3 $\beta$ signaling (Fig. 3B).

To determine whether tatM2NX acts as a competitive antagonist for human TRPM2 channels, we tested the antagonistic capacity of $2 \mu \mathrm{M}$ tatM $2 \mathrm{NX}$ in the presence of $500 \mu \mathrm{M}$ ADPR. In these ligand-saturating conditions, $\mathrm{ADPR}_{\mathrm{i}}$ was similar in control $(173.1 \pm 45.22 \mathrm{pA} / \mathrm{pF}, n=4)$ and in the presence of $2 \mu \mathrm{M}$ tatM2NX (193.4 $\pm 78.00 \mathrm{pA} / \mathrm{pF}, n=4)$ (Fig. 3C). Interestingly, $2 \mu \mathrm{M}$ tatM2NX failed to inhibit $\mathrm{ADPR}_{\mathrm{f}}$ current density $(89.22 \pm 42.22 \mathrm{pA} / \mathrm{pF}, n=4 ; P>0.05)$ compared with control $(117.0 \pm 10.53, n=4)$, indicating that higher concentrations of ADPR can prevent tatM2NX antagonism (Fig. 3C). These results indicate that tatM2NX is a competitive antagonist of TRPM2 and validate the results of our molecular modeling simulations.

TatM2NX C Terminus is Sufficient to Antagonize TRPM2 in Cells. Based on the initial molecular modeling of tatM2NX in complex with TRPM2, we identified two bulky hydrophobic residues, tryptophan in position 33 and valine in position 34 (W33, V34), within the Cterm that remained buried within the ADPR binding site throughout the molecular modeling simulation. We hypothesized that mutating these two residues would potentially abrogate the binding of tatM2NX to the ADPR binding site. Molecular modeling of tatM2NX with W33A and V34A mutations to W33V34 (tatWV-AA) indicated a loss of interaction with the ADPR binding site in any of the top three scoring interaction clusters (Supplemental Fig. 2A), nor did we observe any substantive interactions with this site in any of the lowerscoring results (data not shown). We performed additional computational simulations to assess the capacity of a truncated form of tatM2NX (tat Cterm, containing the last 23 amino acids) to preserve these interactions with the ADPR binding site. The peptide tat Cterm interacts with the ADPR binding site similarly to the parent peptide tatM2NX (Supplemental 
A

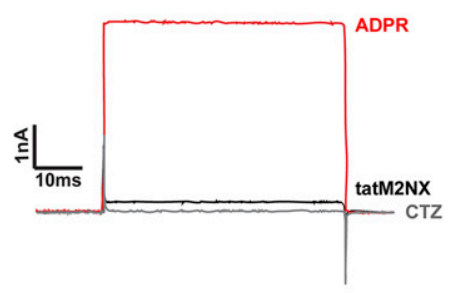

B

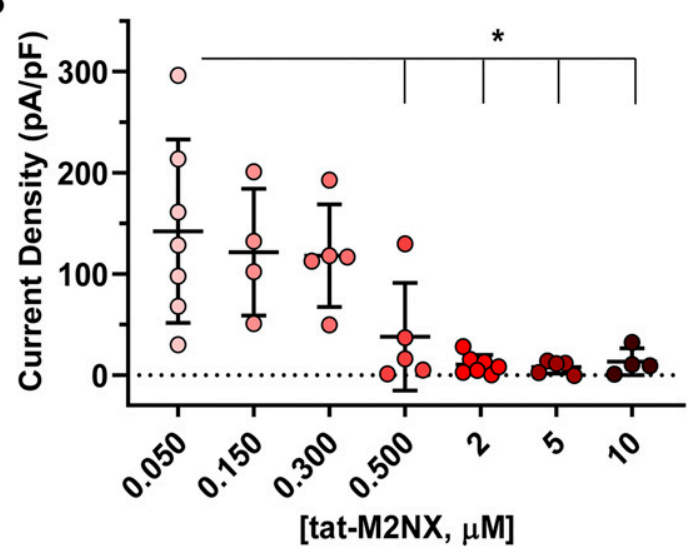

C

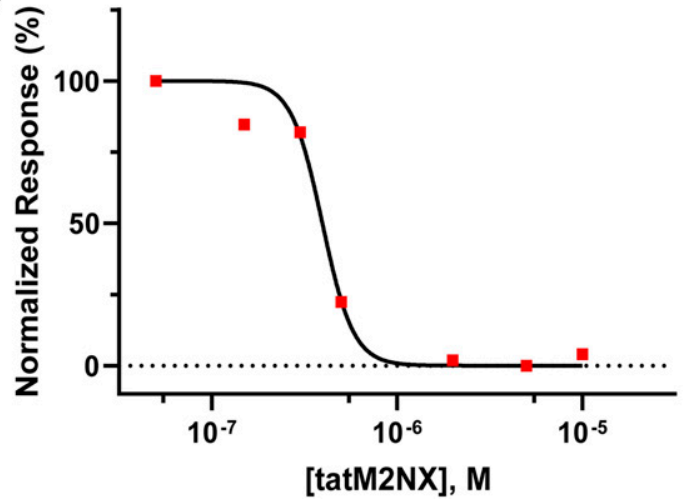

Fig. 2. The peptide tatM2NX inhibits TRPM2 currents in a concentrationdependent manner. (A) Representative TRPM2 initial currents $\left(A D P R_{i}\right.$, 3 to $4 \mathrm{nA}$ ) activated by $100 \mu \mathrm{M}$ ADPR (red), with $2 \mu \mathrm{M}$ tatM2NX (black) or $20 \mu \mathrm{M}$ CTZ (gray). (B) TRPM2 current density at $0.15-10 \mu \mathrm{M}$ tatM2NX compared with TRPM2 current at $0.05 \mu \mathrm{M}$ tatM2NX (ineffective concentration control). (C) Dose-response curve showing normalized response (current density) vs. tatM2NX concentration. Potency was determined normalizing each concentration to TRPM2 current density with $0.05 \mu \mathrm{M}$ tatM2NX. All data are represented as means \pm S.D., and significance $(*)$ was established at $P<0.05$ for $n \geq 4-7$ (at least three experimental days per condition) using one-way ANOVA.

Fig. 2, A and C). This suggested that the $\mathrm{C}$ terminus of tatM2NX was sufficient to interact with the ADPR binding site and would likely recapitulate the inhibitory effects of the parent peptide tatM2NX on TRPM2. Similar to tatM2NX, the tat Cterm peptide was predicted to maintain and enhance interactions with the ADPR binding site over the simulation period (Supplemental Fig. 2, B and C; Supplemental Table 1). In contrast, the interactions of the tatWV-AA peptide did not appear stable and had substantial dissociation from TRPM2 (Supplemental Fig. 2, B and D), suggesting that tatWV-AA lacks physiologically relevant interactions with TRPM2. The overall structure of both tat Cterm and tatWV-AA peptides was highly similar in terms of secondary/tertiary structure (Supplemental Fig. 2B).
Our molecular modeling predicted that stable interactions of tat Cterm and mutating W33V34 to AA would result in a loss of interaction with the ADPR binding site. To validate the molecular modeling results, we performed electrophysiology and cell-based functional assays using $\mathrm{Ca}^{2+}$ imaging. In whole-cell patch clamp, the peptide tatWV-AA contained within the $\mathrm{C}$ terminus (Table 1) lacks antagonism on TRPM2 (116.3 \pm $66.90 \mathrm{pA} / \mathrm{pF}, n=6 ; P>0.05)$ compared with $\mathrm{ADPR}_{\mathrm{f}}(89.39 \pm$ $32.38 \mathrm{pA} / \mathrm{pF}, n=5$ ), indicating that either/both residues are required to confer antagonism (Fig. 4A). Because the tat Cterm peptide clustered by the ADPR binding site, we tested $\mathrm{N}$ and $\mathrm{C}$ termini truncated peptides (23 amino acids each) to determine the extent of antagonism by these truncated forms of tatM2NX (Table 1). In whole-cell patch clamp experiments, tat Cterm displayed significant antagonism $(15.56 \pm 7.52 \mathrm{pA} / \mathrm{pF}, n=6$; $P<0.05)$ compared with $\mathrm{ADPR}_{\mathrm{f}}$ (Fig. $\left.4 \mathrm{~A}\right)$. The $\mathrm{N}$ terminus truncation (tat Nterm) did not inhibit TRPM2 $(141.2 \pm 31.47 \mathrm{pA} / \mathrm{pF}$, $n=4 ; P<0.05)$ compared with $\mathrm{ADPR}_{\mathrm{f}}$ (Fig. $\left.4 \mathrm{~A}\right)$. These results

A

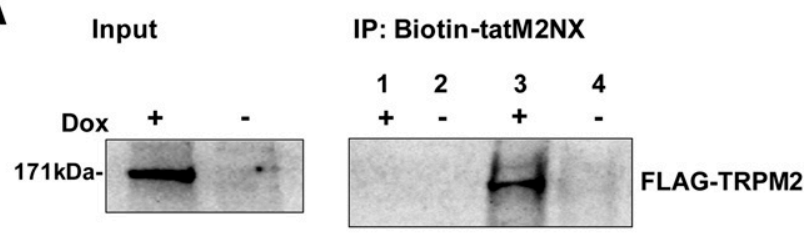

B
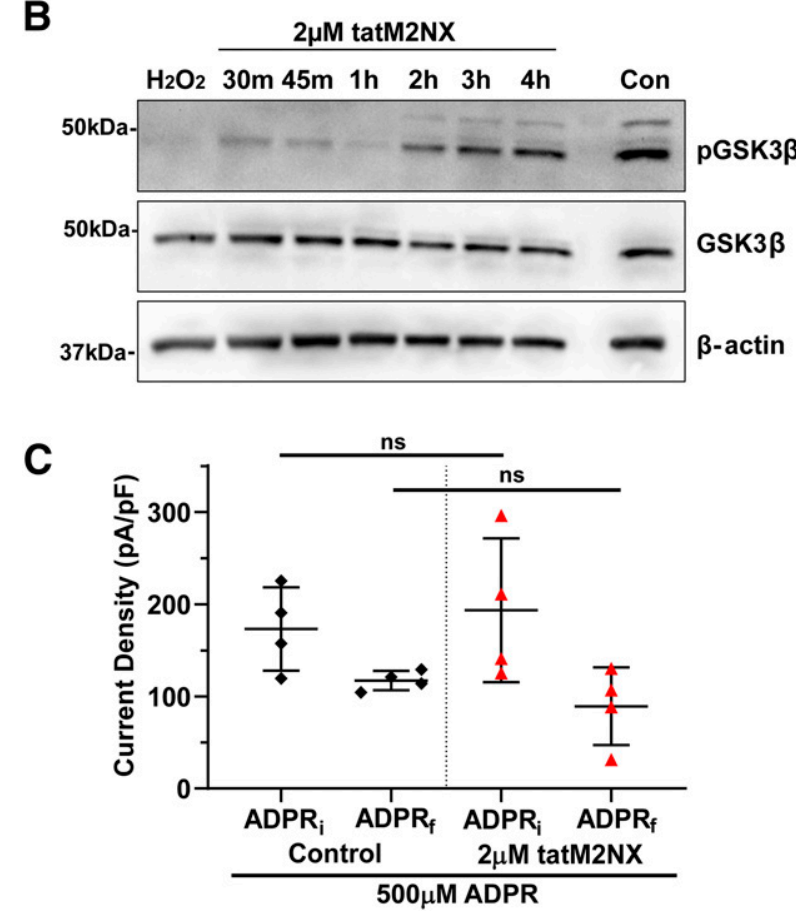

Fig. 3. TatM2NX inhibits TRPM2-mediated GSK3 $\beta$ signaling and competes with ADPR to antagonize TRPM2. (A) Coimmunoprecipitation of biotin-tagged tatM2NX with FLAG-tagged TRPM2 in Dox-inducible HEK293 cells ( + Dox). No biotin-tatM2NX was observed in uninduced cells (-Dox). At least three independent experimental days $(n=3)$. (B) Western blot of HEK293 cells expressing TRPM2 treated with $250 \mu \mathrm{M}$ $\mathrm{H}_{2} \mathrm{O}_{2}$ compared with untreated cells (Con) and cells preincubated with 2 $\mu \mathrm{M}$ tatM2NX for 30 minutes to 4 hours followed by $\mathrm{H}_{2} \mathrm{O}_{2}$ stimulation (10 minutes). (C) $\mathrm{ADPR}_{\mathrm{f}}$ (control) currents with $500 \mu \mathrm{M}$ ADPR in the presence of 0 or $2 \mu \mathrm{M}$ tatM2NX. No significant differences (ns) in $\mathrm{ADPR}_{\mathrm{i}}$ for control and $2 \mu \mathrm{M}$ tatM2NX. All data are represented as means \pm S.D., and significance was established at $P<0.05$ for $n \geq 4$ (at least three experimental days per condition) using Student's $t$ test. Con, untreated control cells; Dox, doxycycline. 
suggest that tatM2NX Cterm is sufficient to inhibit TRPM2, whereas the Nterm alone lacks antagonistic effect. Also, these confirm that the tat-HIV tag, and not the $\mathrm{N}$ terminus sequence, is responsible for direct interactions with TRPM2 and requires the $\mathrm{C}$ terminus residues W33V34 in proximity to the ADPR binding site. Lastly, all $\mathrm{ADPR}_{\mathrm{i}}$ currents were similar in the presence of peptides compared with control (Supplemental Fig. 3A)

To verify that tatM2NX was cell permeable, we incubated biotin-tatM2NX with HEK293 cells for 1 hour, followed by immunocytochemistry for N-terminal tagged biotin-tatM2NX and N-terminal tagged FLAG-TRPM2 within HEK293 cells (Supplemental Fig. 1B). To test whether these peptides show antagonistic effects when applied extracellularly, we measured $\mathrm{Ca}^{2+}$ in $\mathrm{HEK} 293$ cells in response to $\mathrm{H}_{2} \mathrm{O}_{2}$ stimulation. $\mathrm{H}_{2} \mathrm{O}_{2}$ stimulated $\mathrm{Ca}^{2+}$ influx through TRPM2 in the control group $\left(77,441 \pm 34,790 \mathrm{~F} / \mathrm{F}_{0} \times \min ; n=53\right)$ as previously described (Herson et al., 1999; Kraft et al., 2004; Olah et al., 2009; Shimizu et al., 2016) (Fig. 4, B and C). TatM2NX, tat Cterm, and CTZ significantly decreased $\mathrm{Ca}^{2+}$ fluorescence $\left(\mathrm{F} / \mathrm{F}_{0}\right)$ after 20 minutes $\left(47,904 \pm 31,245 \mathrm{~F} / \mathrm{F}_{0} \times \min ; n=48, P<0.05\right.$; $50,600 \pm 26,993 \mathrm{~F} / \mathrm{F}_{0} \times \min ; n=49, P<0.05 ; 31,910 \pm 10,880 \mathrm{~F} /$ $\mathrm{F}_{0} \times \min ; n=25, P<0.05$, respectively) (Fig. 4, B-D). However, tat Nterm $\left(67,851 \pm 33,830 \mathrm{~F} / \mathrm{F}_{0} \times \min ; n=48, P>0.05\right)$ had no effect on $\mathrm{Ca}^{2+}$ fluorescence activated by $\mathrm{H}_{2} \mathrm{O}_{2}$ (Fig. $4, \mathrm{C}$ and $\mathrm{D}$ ). On the other hand, $\mathrm{Ca}^{2+}$ fluorescence with tatWV-AA is higher than $\mathrm{H}_{2} \mathrm{O}_{2}\left(99,192 \pm 39,653 \mathrm{~F} / \mathrm{F}_{0} \times \min ; n=52, P<0.05\right)$ (Fig. 4D). All cellular responses in the presence of peptides had similar fluorescent kinetics as $\mathrm{H}_{2} \mathrm{O}_{2}$ stimulation alone $\left(\mathrm{H}_{2} \mathrm{O}_{2}\right.$, $105.27 \pm 16.81$ frames; tatM2NX, $106.48 \pm 18.73$ frames; tat Cterm, $107 \pm 19.06$ frames; tat Nterm, $110.19 \pm 14.07$ frames; tatWV-AA, $102.81 \pm 22.10$ frames) (Supplemental Fig. 3B). These data suggest that tat Cterm inhibits TRPM2 activity in cells at similar levels as tatM2NX and that residues W33V34 are essential for antagonism.

\section{Discussion}

In this study, we evaluated the pharmacological properties of tatM2NX on TRPM2 channels and found evidence of potent inhibition, with an $\mathrm{IC}_{50}$ of approximately $396 \mathrm{nM}$. Furthermore, we identified two residues within tatM2NX C terminus, W33 and V34, necessary for antagonism. Our molecular modeling of TRPM2 (in the closed channel state) and tatM2NX show direct interactions of tatM2NX with the ADPR binding site. These results are consistent with coimmunoprecipitation of tatM2NX and reduce inhibition in the presence of excess ADPR. Recently, cryoelectron miscroscopy structures validated previous TRPM2 mutagenesis studies indicating that the ADPR binding site is contained within NUDT9-H (Shen et al., 2003; Kühn and Lückhoff, 2004; Yu et al., 2017; Huang et al., 2018; Wang et al., 2018). TRPM2 undergoes conformational changes in the $\mathrm{C}$ terminus NUDT9$\mathrm{H}$ domain, priming an open-channel state upon ADPR binding. Our results support that tatM2NX disrupts the ability of ADPR to open TRPM2 channels. This is the first characterization, to our knowledge, of a peptide inhibitor targeting TRPM2 with in vivo applications.

The tatM2NX peptide was predicted to both directly interact, through its $\mathrm{C}$ terminus, with the human ADPR binding site and to remain wedged in place via the interactions of the tat-HIV (permeability sequence) outside of the ADPR site. Activation of TRPM2 involves extensive conformational changes to the cytosolic $\mathrm{N}$ - and $\mathrm{C}$-terminal regions of TRPM2 that bring the globular domains in closer proximity to one another (Huang et al., 2018; Wang et al., 2018). The
A

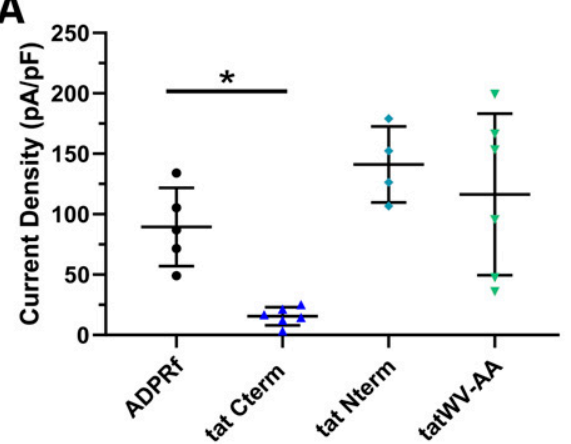

C

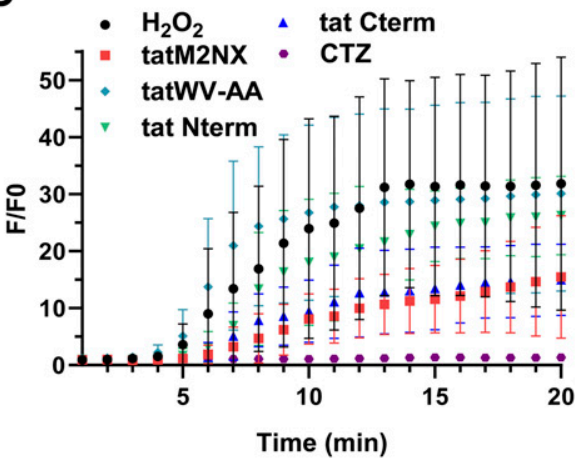

B

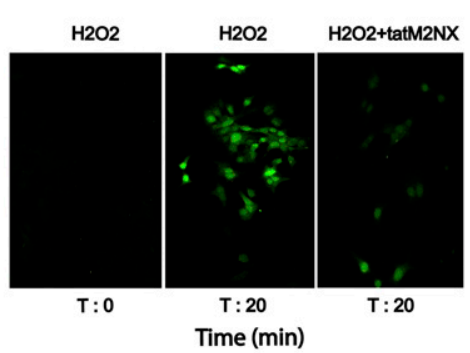

D

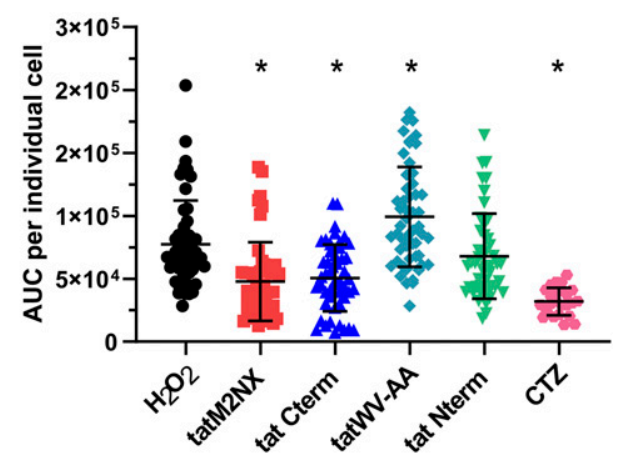

Fig. 4. The $\mathrm{C}$ terminus of tatM2NX is sufficient to antagonize TRPM2. (A) TRPM2 current density in the presence of $2 \mu \mathrm{M}$ tat Cterm (blue), tatWV-AA (sky blue), or tat Nterm (green). All peptides were individually compared with control group $\left(\mathrm{ADPR}_{\mathrm{f}}\right)$. (B) Representative images for $\mathrm{Ca}^{2+}$ imaging experiments at time (T) 0 and 20 minutes for $250 \mu \mathrm{M} \mathrm{H}_{2} \mathrm{O}_{2}$ (control) or $\mathrm{H}_{2} \mathrm{O}_{2}+2 \mu \mathrm{M}$ tatM2NX. Fluo5F, acetoxymethyl (5 $\mu \mathrm{M}$, green) is the fluorescent $\mathrm{Ca}^{2+}$ indicator. (C) Quantification of changes in fluorescence/baseline fluorescence $\left(\mathrm{F} / \mathrm{F}_{0}\right)$ for each peptide $(2 \mu \mathrm{M})$. TatM2NX and tat Cterm significantly inhibit TRPM2 activity in HEK293 cells. $\mathrm{F} / \mathrm{F}_{0}$, corresponding to TRPM2 activity, is inhibited by $20 \mu \mathrm{M}$ CTZ (positive control). (D) Area under the curve (AUC) analysis for each peptide: $\mathrm{H}_{2} \mathrm{O}_{2}$ control (black), tatM2NX (red), tat Cterm (blue), tatWV-AA (sky blue), tat Nterm (green). tatM2NX, tat Cterm, and CTZ significantly decreased AUC for TRPM2 activity, whereas tatWV-AA significantly increased AUC. All data are represented as means \pm S.D., and significance $(*)$ was established as $P<0.05$ using one-way ANOVA with Dunnett's post hoc for multiple group comparison, $n \geq 4-10$ for electrophysiology, and $n \geq 25-53$ cells for $\mathrm{Ca}^{2+}$ imaging (four to six independent experiments). 
modeling results suggest that, in addition to occluding the ADPR binding site, tatM2NX may also directly impede the molecular mechanics of activation by blocking or impairing these conformational changes.

The interactions of tat Cterm with the ADPR binding site were stable, and the peptide itself moved further into the ADPR binding site over the simulation period. These results suggest that the $\mathrm{C}$ terminus hydrophobic interactions with the ADPR binding site are sufficient to maintain the TRPM2tat Cterm interactions. Interestingly, the best-predicted complexes for the active inhibitory peptides involved binding at the human ADPR site with extensive stable interactions (Supplemental Table 1). Future mutagenesis studies may reveal which interactions are required for inhibition. Other top-ranked complexes for both tatM2NX and tat Cterm peptides involved interaction with the region corresponding to the ADPR binding site in zebrafish TRPM2 (Fliegert et al., 2018; Huang et al., 2018), suggesting that these peptides may also have inhibitory effects against the activity of zebrafish TRPM2. Also, we have shown efficacy in mouse models of brain ischemia (Shimizu et al., 2016; Dietz et al., 2019); therefore, this tool has potential use in multiple systems and species.

Isolating TRPM2 function in physiology and pathophysiology is critical to understand signaling downstream of TRPM2 channel activation. Previous studies used nonspecific pharmacological inhibitors to show the involvement of TRPM2 function increasing oxidative stress signaling (Fonfria et al., 2004; Perraud et al., 2005). More recently, total genetic ablation approaches in rodents associate TRPM2 with mood disorders, social interactions, temperature control, and Alzheimer disease, implicating increased TRPM2 channel activity to various neurological diseases across many brain regions (Jang et al., 2015; Ostapchenko et al., 2015; Tan and McNaughton, 2016; Alawieyah Syed Mortadza et al., 2018; Andoh et al., 2019). Therefore, pharmacological targeting of this channel has the potential to provide clinical benefit in several neurological disorders. However, there are no available inhibitors that would be amenable to clinical use. We believe tatM2NX represents the most promising TRPM2 pharmacological inhibitor available to date. A single dose of tatM2NX effectively crosses the blood-brain barrier (BBB) while providing TRPM2 specific neuroprotection in stroke and cardiac arrest animals, setting precedence for the use of tatM2NX in vivo (Shimizu et al., 2016; Dietz et al., 2019). These studies demonstrate the ability of tatM2NX to cross the $\mathrm{BBB}$ and impact brain injury, consistent with several previous studies showing tat-conjugated proteins crossing the BBB (Cao et al., 2002; Hill et al., 2012; Lalatsa et al., 2014; Rizzuti et al., 2015). Furthermore, our recent study in cardiac arrest implicated TRPM2 channel inhibition of GSK3 $\beta$ in longterm functional deficits (Dietz et al., 2019). Consistent with this, data presented here show that tatM2NX prevents oxidative stress-induced activation (dephosphorylation) of GSK3 $\beta$. In ex vivo experiments with the human TRPM2 channel, tatM2NX inhibits with similar high potency as JNJ-28583113 (Fourgeaud et al., 2019) and scalaradial, another potent TRPM2 antagonist that also targets TRPM7 (Starkus et al., 2017). We assessed specificity of tatM2NX for TRPM2 (Shimizu et al., 2016) by showing no additional efficacy in TRPM2-/- mice on neuroprotection following stroke. Additionally, TRPM2 expression in other organs magnifies the use of tatM2NX in a variety of disease models (Lange et al., 2009; Park et al., 2016; Almasi et al., 2018). Future studies will determine the reversibility and specificity of tatM2NX. Furthermore, comparison of binding pockets of TRPM channels 4 , 7 , and 8 indicates that the ADPR binding site on TRPM2 is different (Supplemental Fig. 4). Future studies will address off-target effects and confirm the molecular modeling performed for TRPM channel members and other proteins.

TatM2NX is a cell-permeable inhibitor with high potency that makes extensive noncovalent interactions and favorable contacts with a number of residues of the ADPR binding site within the NUDT9-H domain. Many of these interactions were predicted and maintained in the tat Cterm peptide. In contrast, there were no predicted interactions between tatWV-AA and the ADPR binding site. Furthermore, both tatM2NX and tat Cterm exhibited competitive inhibition in cells, providing validation of the in silico predictions. Taken together, these data provide a mechanistic rationale for the observed inhibition of TRPM2 channels. Because of the technical constraints of our experiments (see Materials and Methods section), we were not able to assess the kinetics of inhibition or possible reversibility of tatM2NX. Our data indicate that tatM2NX is a competitive antagonist, leading us to speculate that tatM2NX is likely a reversible inhibitor binding at the ADPR binding site. Further experiments are needed to determine the reversal kinetics of this cell-permeable TRPM2 channel antagonist. Nonetheless, our in vivo data (Shimizu et al., 2016; Dietz et al., 2019) show effective inhibition of TRPM2 channels in the intact brain, making this a valuable research tool for the study of TRPM2 channels. Beyond the characterization of tatM2NX as a novel TRPM2 antagonist, our aim is to provide a research tool that will move the field of TRP channels forward and aid in specifically studying TRPM2 molecular mechanisms in cell physiology and pathophysiology of diseases. The peptide tatM2NX will serve as a potential source of a new generation of derivatives that may be more potent and specific at antagonizing TRPM2.

\section{Acknowledgments}

We thank Dr. Anne Perraud for providing HEK293 cells containing an N-terminal FLAG epitope tag in a modified pCDNA4/TO vector. Special thanks to the Computational Chemistry and Biology Core Facility at the University of Colorado Anschutz Medical Campus for assistance with the structural modeling and simulation studies.

\section{Authorship Contributions}

Participated in research design: Cruz-Torres, Backos, Herson. Conducted experiments: Cruz-Torres, Backos.

Contributed new reagents or analytic tools: Cruz-Torres, Backos, Herson.

Performed data analysis: Cruz-Torres, Backos, Herson.

Wrote or contributed to the writing of the manuscript: Cruz-Torres, Backos, Herson.

\section{References}

Alawieyah Syed Mortadza S, Sim JA, Neubrand VE, and Jiang LH (2018) A critical role of TRPM2 channel in $A \beta_{42}$-induced microglial activation and generation of tumor necrosis factor- $\alpha$. Glia 66:562-575.

Alim I, Teves L, Li R, Mori Y, and Tymianski M (2013) Modulation of NMDAR subunit expression by TRPM2 channels regulates neuronal vulnerability to ischemic cell death. J Neurosci 33:17264-17277.

Almasi S, Kennedy BE, El-Aghil M, Sterea AM, Gujar S, Partida-Sánchez S, and El Hiani Y (2018) TRPM2 channel-mediated regulation of autophagy maintains mitochondrial function and promotes gastric cancer cell survival via the JNKsignaling pathway. J Biol Chem 293:3637-3650.

Andoh C, Nishitani N, Hashimoto E, Nagai Y, Takao K, Miyakawa T, Nakagawa T, Mori Y, Nagayasu K, Shirakawa H, et al. (2019) TRPM2 confers 
susceptibility to social stress but is essential for behavioral flexibility. Brain Res 1704:68-77.

Bai JZ and Lipski J (2010) Differential expression of TRPM2 and TRPV4 channels and their potential role in oxidative stress-induced cell death in organotypic hippocampal culture. Neurotoxicology 31:204-214.

Brizzard BL, Chubet RG, and Vizard DL (1994) Immunoaffinity purification of FLAG epitope-tagged bacterial alkaline phosphatase using a novel monoclonal antibody and peptide elution. Biotechniques 16:730-735.

Cao G, Pei W, Ge H, Liang Q, Luo Y, Sharp FR, Lu A, Ran R, Graham SH, and Chen J (2002) In vivo delivery of a Bcl-xL fusion protein containing the TAT protein transduction domain protects against ischemic brain injury and neuronal apoptosis. J Neurosci 22:5423-5431.

Chen GL, Zeng B, Eastmond S, Elsenussi SE, Boa AN, and Xu SZ (2012) Pharmacological comparison of novel synthetic fenamate analogues with econazole and 2-APB on the inhibition of TRPM2 channels. Br J Pharmacol 167:1232-1243.

Chen R, Li L, and Weng Z (2003) ZDOCK: an initial-stage protein-docking algorithm. Proteins 52:80-87.

Chung KK, Freestone PS, and Lipski J (2011) Expression and functional properties of TRPM2 channels in dopaminergic neurons of the substantia nigra of the rat. $J$ Neurophysiol 106:2865-2875.

Dietz RM, Cruz-Torres I, Orfila JE, Patsos OP, Shimizu K, Chalmers N, Deng G, Tiemeier E, Quillinan N, and Herson PS (2019) Reversal of global ischemia-induced cognitive dysfunction by delayed inhibition of TRPM2 ion channels. Transl Stroke Res DOI: 10.1007/s12975-019-00712-z [published ahead of print].

Fliegert R, Bauche A, Wolf Pérez AM, Watt JM, Rozewitz MD, Winzer R, Janus M, Gu F, Rosche A, Harneit A, et al. (2017a) 2'-Deoxyadenosine 5'-diphosphoribose is an endogenous TRPM2 superagonist. Nat Chem Biol 13:1036-1044.

Fliegert R, Hölzer HT, and Guse AH (2018) TRPM2 activation: paradigm shifted? Cell Calcium 76:132-134.

Fliegert R, Watt JM, Schöbel A, Rozewitz MD, Moreau C, Kirchberger T, Thomas MP, Sick W, Araujo AC, Harneit A, et al. (2017b) Ligand-induced activation of human TRPM2 requires the terminal ribose of ADPR and involves Arg1433 and Tyr1349. Biochem J 474:2159-2175.

Fonfria E, Marshall IC, Benham CD, Boyfield I, Brown JD, Hill K, Hughes JP, Skaper SD, and McNulty S (2004) TRPM2 channel opening in response to oxidative stress is dependent on activation of poly(ADP-ribose) polymerase. $\mathrm{Br}$ J Pharmacol 143:186-192.

Fonfria E, Murdock PR, Cusdin FS, Benham CD, Kelsell RE, and McNulty S (2006) Tissue distribution profiles of the human TRPM cation channel family. $J$ Recept Signal Transduct Res 26:159-178.

Fourgeaud L, Dvorak C, Faouzi M, Starkus J, Sahdeo S, Wang Q, Lord B, Coate H, Taylor N, He Y, et al. (2019) Pharmacology of JNJ-28583113: a novel TRPM2 antagonist. Eur J Pharmacol 853:299-307.

Gelderblom M, Melzer N, Schattling B, Göb E, Hicking G, Arunachalam P, Bittner S Ufer F, Herrmann AM, Bernreuther C, et al. (2014) Transient receptor potential melastatin subfamily member 2 cation channel regulates detrimental immune cell invasion in ischemic stroke. Stroke 45:3395-3402.

Hantute-Ghesquier A, Haustrate A, Prevarskaya N, and Lehen'kyi V (2018) TRPM family channels in cancer. Pharmaceuticals (Basel) 11:E58.

Haraguchi K, Kawamoto A, Isami K, Maeda S, Kusano A, Asakura K, Shirakawa H, Mori Y, Nakagawa T, and Kaneko S (2012) TRPM2 contributes to inflammatory and neuropathic pain through the aggravation of pronociceptive inflammatory responses in mice. $J$ Neurosci 32:3931-3941.

Heiner I, Eisfeld J, Warnstedt M, Radukina N, Jüngling E, and Lückhoff A (2006) Endogenous ADP-ribose enables calcium-regulated cation currents through TRPM2 channels in neutrophil granulocytes. Biochem $J$ 398:225-232.

Herson PS, Dulock KA, and Ashford ML (1997) Characterization of a nicotinamideadenine dinucleotide-dependent cation channel in the CRI-G1 rat insulinoma cell line. J Physiol 505:65-76.

Herson PS, Lee K, Pinnock RD, Hughes J, and Ashford ML (1999) Hydrogen peroxide induces intracellular calcium overload by activation of a non-selective cation channel in an insulin-secreting cell line. J Biol Chem 274:833-841.

Hess B, Bekker B, Berendsen HJC, and Fraaije JGEM (1997) LINCS: a linear constraint solver for molecular simulations. J Comput Chem 18:1463-1472.

Hill K, McNulty S, and Randall AD (2004) Inhibition of TRPM2 channels by the antifungal agents clotrimazole and econazole. Naunyn Schmiedebergs Arch Pharmacol 370:227-237.

Hill MD, Martin RH, Mikulis D, Wong JH, Silver FL, Terbrugge KG, Milot G, Clark WM, Macdonald RL, Kelly ME, et al.; ENACT Trial Investigators (2012) Safety and efficacy of NA-1 in patients with iatrogenic stroke after endovascular aneurysm repair (ENACT): a phase 2, randomised, double-blind, placebo-controlled trial. Lancet Neurol 11:942-950.

Hoffman NE, Miller BA, Wang J, Elrod JW, Rajan S, Gao E, Song J, Zhang XQ, Hirschler-Laszkiewicz I, Shanmughapriya S, et al. (2015) $\mathrm{Ca}^{2+}$ entry via Trpm2 is essential for cardiac myocyte bioenergetics maintenance. Am J Physiol Heart Circ Physiol 308:H637-H650

Hooft RW, Vriend G, Sander C, and Abola EE (1996) Errors in protein structures. Nature 381:272.

Huang Y, Winkler PA, Sun W, Lü W, and Du J (2018) Architecture of the TRPM2 channel and its activation mechanism by ADP-ribose and calcium. Nature 562:145-149.

Inamura K, Sano Y, Mochizuki S, Yokoi H, Miyake A, Nozawa K, Kitada C, Matsushime H, and Furuichi K (2003) Response to ADP-ribose by activation of TRPM2 in the CRI-G1 insulinoma cell line. J Membr Biol 191:201-207.

Iordanov I, Mihályi C, Tóth B, and Csanády L (2016) The proposed channel-enzyme transient receptor potential melastatin 2 does not possess ADP ribose hydrolase activity. eLife 5:e17600.

Jakalian A, Jack DB, and Bayly CI (2002) Fast, efficient generation of high-quality atomic charges. AM1-BCC model: II. Parameterization and validation. J Comput Chem 23:1623-1641.
Jang Y, Lee SH, Lee B, Jung S, Khalid A, Uchida K, Tominaga M, Jeon D, and Oh U (2015) TRPM2, a susceptibility gene for bipolar disorder, regulates glycogen synthase kinase-3 activity in the brain. $J$ Neurosci 35:11811-11823.

Kraft R, Grimm C, Grosse K, Hoffmann A, Sauerbruch S, Kettenmann H, Schultz G, and Harteneck C (2004) Hydrogen peroxide and ADP-ribose induce TRPM2mediated calcium influx and cation currents in microglia. Am J Physiol Cell Physiol 286:C129-C137.

Krieger E, Darden T, Nabuurs SB, Finkelstein A, and Vriend G (2004) Making optimal use of empirical energy functions: force-field parameterization in crysta space. Proteins 57:678-683.

Krieger E, Dunbrack RL Jr, Hooft RW, and Krieger B (2012) Assignment of protonation states in proteins and ligands: combining pKa prediction with hydrogen bonding network optimization. Methods Mol Biol 819:405-421.

Krieger E, Joo K, Lee J, Lee J, Raman S, Thompson J, Tyka M, Baker D, and Karplus $\mathrm{K}$ (2009) Improving physical realism, stereochemistry, and side-chain accuracy in homology modeling: four approaches that performed well in CASP8. Proteins $\mathbf{7 7}$ (Suppl 9):114-122.

Krieger E, Koraimann G, and Vriend G (2002) Increasing the precision of comparative models with YASARA NOVA--a self-parameterizing force field. Proteins 47: 393-402.

Krieger E, Nielsen JE, Spronk CA, and Vriend G (2006) Fast empirical pKa prediction by Ewald summation. $J$ Mol Graph Model 25:481-486.

Krieger E and Vriend G (2015) New ways to boost molecular dynamics simulations. $J$ Comput Chem 36:996-1007.

Kühn FJ and Lückhoff A (2004) Sites of the NUDT9-H domain critical for ADP-ribose activation of the cation channel TRPM2. J Biol Chem 279:46431-46437.

Lalatsa A, Schatzlein AG, and Uchegbu IF (2014) Strategies to deliver peptide drugs to the brain. Mol Pharm 11:1081-1093.

Lange I, Yamamoto S, Partida-Sanchez S, Mori Y, Fleig A, and Penner R (2009) TRPM2 functions as a lysosomal Ca2+-release channel in beta cells. Sci Signal 2 : ra23.

Li L, Chen R, and Weng Z (2003) RDOCK: refinement of rigid-body protein docking predictions. Proteins 53:693-707.

Li X and Jiang LH (2019) A critical role of the transient receptor potential melastatin 2 channel in a positive feedback mechanism for reactive oxygen species-induced delayed cell death. J Cell Physiol 234:3647-3660.

Maier JA, Martinez C, Kasavajhala K, Wickstrom L, Hauser KE, and Simmerling C (2015) ff14SB: improving the accuracy of protein side chain and backbone parameters from ff99SB. J Chem Theory Comput 11:3696-3713.

McHugh D, Flemming R, Xu SZ, Perraud AL, and Beech DJ (2003) Critical intracellular $\mathrm{Ca} 2+$ dependence of transient receptor potential melastatin 2 (TRPM2) cation channel activation. J Biol Chem 278:11002-11006.

Olah ME, Jackson MF, Li H, Perez Y, Sun HS, Kiyonaka S, Mori Y, Tymianski M, and MacDonald JF (2009) Ca2+-dependent induction of TRPM2 currents in hippocampal neurons. J Physiol 587:965-979.

Ostapchenko VG, Chen M, Guzman MS, Xie YF, Lavine N, Fan J, Beraldo FH Martyn AC, Belrose JC, Mori Y, et al. (2015) The transient receptor potential melastatin 2 (TRPM2) channel contributes to $\beta$-amyloid oligomer-related neurotoxicity and memory impairment. $J$ Neurosci 35:15157-15169.

Park YR, Chun JN, So I, Kim HJ, Baek S, Jeon JH, and Shin SY (2016) Data-driven analysis of TRP channels in cancer: linking variation in gene expression to clinical significance. Cancer Genomics Proteomics 13:83-90.

Perraud AL, Fleig A, Dunn CA, Bagley LA, Launay P, Schmitz C, Stokes AJ, Zhu Q, Bessman MJ, Penner R, et al. (2001) ADP-ribose gating of the calciumpermeable LTRPC 2 channel revealed by Nudix motif homology. Nature 411 $595-599$

Perraud AL, Shen B, Dunn CA, Rippe K, Smith MK, Bessman MJ, Stoddard BL, and Scharenberg AM (2003) NUDT9, a member of the Nudix hydrolase family, is an evolutionarily conserved mitochondrial ADP-ribose pyrophosphatase. J Biol Chem 278:1794-1801.

Perraud AL, Takanishi CL, Shen B, Kang S, Smith MK, Schmitz C, Knowles HM, Ferraris D, Li W, Zhang J, et al. (2005) Accumulation of free ADP-ribose from mitochondria mediates oxidative stress-induced gating of TRPM2 cation channels. $J$ Biol Chem 280:6138-6148.

Pierce B and Weng Z (2007) ZRANK: reranking protein docking predictions with an optimized energy function. Proteins 67:1078-1086.

Rizzuti M, Nizzardo M, Zanetta C, Ramirez A, and Corti S (2015) Therapeutic applications of the cell-penetrating HIV-1 Tat peptide. Drug Discov Today 20: $76-85$

Rueden CT, Schindelin J, Hiner MC, DeZonia BE, Walter AE, Arena ET, and Eliceiri KW (2017) Image J2: ImageJ for the next generation of scientific image data. BMC Bioinformatics 18:529.

Ryan K, Backos DS, Reigan P, and Patel M (2012) Post-translational oxidative modification and inactivation of mitochondrial complex I in epileptogenesis. $J$ Neurosci 32:11250-11258.

Shen BW, Perraud AL, Scharenberg A, and Stoddard BL (2003) The crystal structure and mutational analysis of human NUDT9. J Mol Biol 332:385-398.

Shimizu T, Dietz RM, Cruz-Torres I, Strnad F, Garske AK, Moreno M, Venna VR, Quillinan N, and Herson PS (2016) Extended therapeutic window of a novel peptide inhibitor of TRPM2 channels following focal cerebral ischemia. Exp Neurol $\mathbf{2 8 3}$ (Pt A):151-156.

Shimizu T, Macey TA, Quillinan N, Klawitter J, Perraud AL, Traystman RJ, and Herson PS (2013) Androgen and PARP-1 regulation of TRPM2 channels after ischemic injury. $J$ Cereb Blood Flow Metab 33:1549-1555.

Smith MA, Herson PS, Lee K, Pinnock RD, and Ashford ML (2003) Hydrogen-peroxide-induced toxicity of rat striatal neurones involves activation of a non-selective cation channel. J Physiol 547:417-425.

Smith N, Bornikova L, Noetzli L, Guglielmone H, Minoldo S, Backos DS, Jacobson L, Thornburg CD, Escobar M, White-Adams TC, et al. (2018) Identification and characterization of novel mutations implicated in congenital fibrinogen disorders. Res Pract Thromb Haemost 2:800-811. 
Starkus JG, Poerzgen P, Layugan K, Kawabata KG, Goto JI, Suzuki S, Myers G, Kelly M, Penner R, Fleig A, et al. (2017) Scalaradial is a potent inhibitor of transient receptor potential melastatin 2 (TRPM2) ion channels. J Nat Prod 80 $2741-2750$.

Tan CH and McNaughton PA (2016) The TRPM2 ion channel is required for sensitivity to warmth. Nature 536:460-463.

Tong Q, Zhang W, Conrad K, Mostoller K, Cheung JY, Peterson BZ, and Miller BA (2006) Regulation of the transient receptor potential channel TRPM2 by the Ca2+ sensor calmodulin. J Biol Chem 281:9076-9085.

Tóth B and Csanády L (2012) Pore collapse underlies irreversible inactivation of TRPM2 cation channel currents. Proc Natl Acad Sci USA 109:13440-13445.

Tóth B, Iordanov I, and Csanády L (2015) Ruling out pyridine dinucleotides as true TRPM2 channel activators reveals novel direct agonist ADP-ribose-2'-phosphate. $J$ Gen Physiol 145:419-430.

Verma S, Quillinan N, Yang YF, Nakayama S, Cheng J, Kelley MH, and Herson PS (2012) TRPM2 channel activation following in vitro ischemia contributes to male hippocampal cell death. Neurosci Lett 530:41-46.

Vriend G (1990) WHAT IF: a molecular modeling and drug design program. J Mol Graph 8:52-56, 29

Wang J, Wolf RM, Caldwell JW, Kollman PA, and Case DA (2004) Development and testing of a general amber force field. J Comput Chem 25:1157-1174.
Wang L, Fu TM, Zhou Y, Xia S, Greka A, and Wu H (2018) Structures and gating mechanism of human TRPM2. Science 362:eaav4809.

Xu D and Zhang Y (2012) Ab initio protein structure assembly using continuous structure fragments and optimized knowledge-based force field. Proteins 80: 1715-1735.

Xu D and Zhang Y (2013) Toward optimal fragment generations for ab initio protein structure assembly. Proteins 81:229-239.

Yu P, Xue X, Zhang J, Hu X, Wu Y, Jiang LH, Jin H, Luo J, Zhang L, Liu Z, et al. (2017) Identification of the ADPR binding pocket in the NUDT9 homology domain of TRPM2. J Gen Physiol 149:219-235.

Zhang H, Liu H, Luo X, Wang Y, Liu Y, Jin H, Liu Z, Yang W, Yu P, Zhang L, et al. (2018) Design, synthesis and biological activities of 2,3-dihydroquinazolin-4(1H) one derivatives as TRPM2 inhibitors. Eur J Med Chem 152:235-252.

Address correspondence to: I. Cruz-Torres, Department of Anesthesiology, University of Colorado School of Medicine, 12800 E. 19th Ave, Aurora, CO 80045. E-mail: ivelisse.cruz-torres@ucdenver.edu; or P.S. Herson, Department of Anesthesiology, University of Colorado School of Medicine, 12800 E. 19th Ave, Aurora, CO 80045. E-mail: paco.herson@ucdenver.edu 